\title{
Paraelectric properties of PbTe doped with Ga
}

\author{
V. Tetyorkin and S. Movchan \\ Institute of Semiconductor Physics of NAS of Ukraine, pr. Nauki, 45, Kyiv, Ukraine, 03028.
}

\begin{abstract}
The static dielectric constant was investigated as a function of temperature and carrier concentration in $\operatorname{In}(\mathrm{Cu}) / p$-PbTe Schottky contacts. Single crystals of PbTe doped with $\mathrm{Ga}$ were used as substrates for preparation of the contacts. The static dielectric constant was found to obey the Curie-Weiss law with the negative Curie temperature for all samples investigated including those ones with the lowest values of the hole concentration. There is an evidence that the Curie temperature is dependent on the compensation state in doped PbTe. The increase of the compensation results in nonmonotonous dependence of the Curie temperature on carrier concentration. The effect of the foreign impurities and native defects on the static dielectric constant and Curie temperature is discussed.
\end{abstract}

Keywords: lead telluride, dielectric constant, Curie temperature, Schottky contacts.

Paper received 26.04.00, revised manuscript received 05.06.00, accepted for publication 30.06.00.

\section{Introduction}

Lead telluride is characterized by variety of unusual lattice and electronic properties at low temperatures. For instance, very large dielectric constant in $\mathrm{PbTe}$ reflects its tendency to the phase transition from $\mathrm{NaCl}$ to rhombohedral lattice structure $[1 \div 5]$. Paraelectric behavior of $\mathrm{PbTe}$ was explained by the softening of TOphonon due to strong interband electron-phonon coupling. The static dielectric constant $\varepsilon_{\mathrm{o}}$ in PbTe was found to obey the Curie-Weiss law with the negative Curie temperature $T_{c}$. There have been many attempts to determine $\varepsilon_{\mathrm{o}}$ using different measurement techniques $[2,3]$. However, experimental values of $\varepsilon_{\mathrm{o}}$ and $T_{c}$ exhibit large discrepancies in literature. These discrepancies can arise from several reasons. Both, the Curie temperature and dielectric constant depend on the concentration of free carriers introduced by the native defects (doubly ionized vacancies of $\mathrm{Pb}$ and Te and singly ionized interstitials of $\mathrm{Pb}$ atoms [6,7]). The fact is known for years native defects create resonant states in the conduction and valence bands of lead telluride and no freeze-out occurs even at very low temperatures [6,7]. As a rule, the concentration of native defects in as-grown bulk crystals as well as epitaxial layers exceeds $10^{18} \mathrm{~cm}^{-3}[1,8]$. Multistep thermal annealing in Te-rich atmosphere allows one to reduce the concentration to approximately $1 \times 10^{17} \mathrm{~cm}^{-3}[8]$. Even when improved crystal growth technique, such as hot-wall epitaxy, was used, the concentration exceeds $1 \times 10^{16} \mathrm{~cm}^{-3}$ [9]. At temperatures $T \leq 78 \mathrm{~K}$ free carriers are degenerated for the values $n, p \geq 10^{17} \mathrm{~cm}^{-3}$. In degenerate $n$-and $p$-type PbTe the dependence of $T_{c}$ on the carrier concentration is correlated with position of the Fermi level in the conduction or valence band (see below). Due to Konsin and Kristofell [10], in nondegenerate semiconductors the Curie temperature should increase with decrease of the carrier concentration. So, one may expect the Curie temperature in $\mathrm{PbTe}$ to be monotonous function of the carrier concentration for both degenerate and nondegenerate crystals.

The doping of PbTe by foreign impurities seems to be the only method which can be used for the purpose of preparation of $\mathrm{PbTe}$ single crystals with low values $n, p \leq 1 \times 10^{16} \mathrm{~cm}^{-3}$ of the carrier concentration. Due to $\mathrm{PbTe}$ has soft and deformable crystal lattice foreign impurities can be introduced to it in large amounts. For instance, shown in Fig. 1 is the variation of the concentration with gallium doping in as-grown $\mathrm{PbTe}$ single crystals [11]. As seen, the crystals of $n$ - and $p$-type conductivity with the carrier concentration $\sim 10^{13} \mathrm{~cm}^{-3}$ can be prepared. Obviously, low values of the carrier concentration result from the compensation of native defects by impurity atoms. One may expect the degree of the compensation in doped with Ga samples is higher in comparison with undoped crystals investigated earlier. This seems to be important result because of, as was suggested 


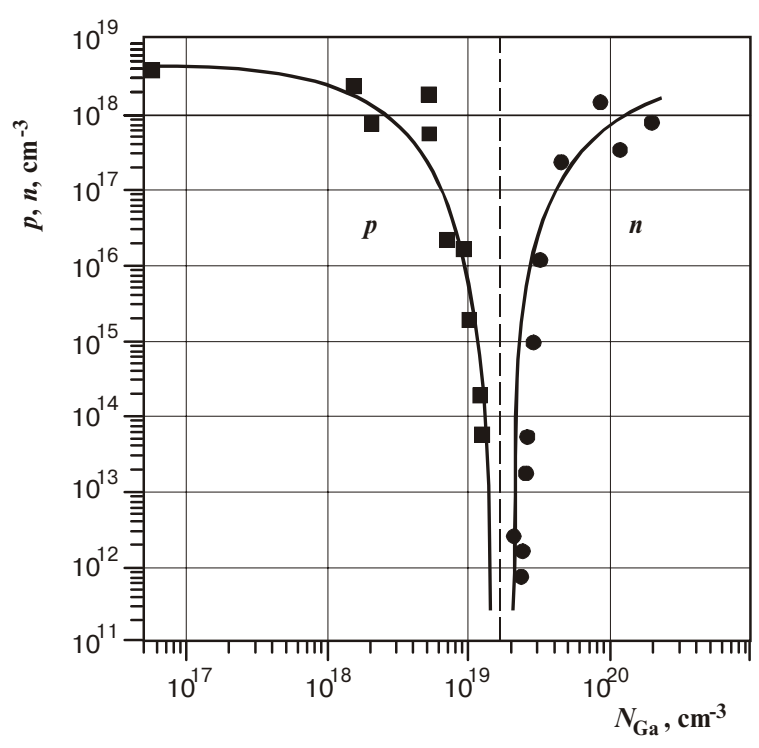

Fig. 1. Free carrier concentration as a function of Ga concentration in PbTe single crystals.

in [12], the Curie temperature may depend on the compensation. Experimental results obtained in the present paper confirm this suggestion.

The aim of this work is to study effect of doping on paraelectric properties of lead telluride at low temperatures.

\section{Experimental details}

Doped with Ga bulk crystals of lead telluride were grown by Czochralski method. The as-grown crystal rods were divided on slices of a thickness $0.8 \div 1.0 \mathrm{~mm}$ followed by mechanical polishing on both sides. Finally, the surface of slices were anode-mechanically polished. The substrates thus prepared were mounted into vacuum chamber for thermal deposition of indium. The contacts $\mathrm{Cu} / p$ $\mathrm{PbTe}$ were prepared by chemical deposition of metal at room temperature to obtain abrupt interface [13]. Mesa structures with an area $A=3 \times 10^{-3} \mathrm{~cm}^{2}$ were delineated by chemical etching. Ohmic contacts to the back side of the substrates were made by soldering of thin copper wires using In-Au-As alloy. The capacitance-voltage characteristics of the contacts were measured in the temperature range $4.2 \div 160 \mathrm{~K}$ using $1 \mathrm{MHz}$ capacitance bridge. Previously, structural and electrical properties of $\mathrm{PbTe}: \mathrm{Ga}$ substrates as well as photoelectrical and electrical properties of the Schottky contacts on their base were investigated in details at temperatures $T \geq 78 \mathrm{~K}[11,13]$.

\section{Results and discussion}

In a contact with abrupt junction the barrier capacitance is given by [14]:
$C=\left(\frac{q \varepsilon_{0} N}{2}\right)^{1 / 2}\left(U_{D}-U-\frac{k T}{q}\right)^{-1 / 2}$,

were $U_{d}$ is the diffusion potential, $U$ is the reverse bias voltage, $N$ is the concentration of ionized impurities. In (1) the dielectric constant is assumed to depend on temperature in accordance with the Curie-Weiss law $\varepsilon_{0}^{-1}=c\left(T-T_{c}\right)$. The reciprocal of the static dielectric constant can be determined from the slope of $C^{-2}=f(U)$ dependence if the value of $N$ is known from independent measurements. For the chemically prepared contacts there is no reason for the hole concentration to be changed from the value measured in the starting material. However, for the contacts prepared by thermal evaporation of indium additional measurements are desirable. For this purpose, differential Hall-effect measurements (in Van der Pauw geometry) have been performed before and after metal deposition procedure. The difference between two measurements was observed in the most compensated material with $p \sim 10^{13} \mathrm{~cm}^{-3}$. Also, the contacts prepared on such substrates exhibit some peculiarities for capacitance- and current-voltage characteristics and will be analyzed elsewhere. Typical measured and calculated capacitance-voltage characteristics plotted as $C^{-2}=f(U)$ are shown in Fig. 2. In the calculation, the dielectric constant served as a fitting parameter.

In Fig. 3 the reciprocal dielectric constant is shown as a function of temperature for two samples. Experimental data were obtained for reverse bias voltage $-0.1 \mathrm{~V}$. As seen, they are well approximated by straight lines at $T \geq 40 \mathrm{~K}$. From the intercept with temperature axis the values of the Curie temperature was derived. The dependence of $T_{c}$ on the hole concentration is shown in Fig. 4. At least two features should be pointed out for the data

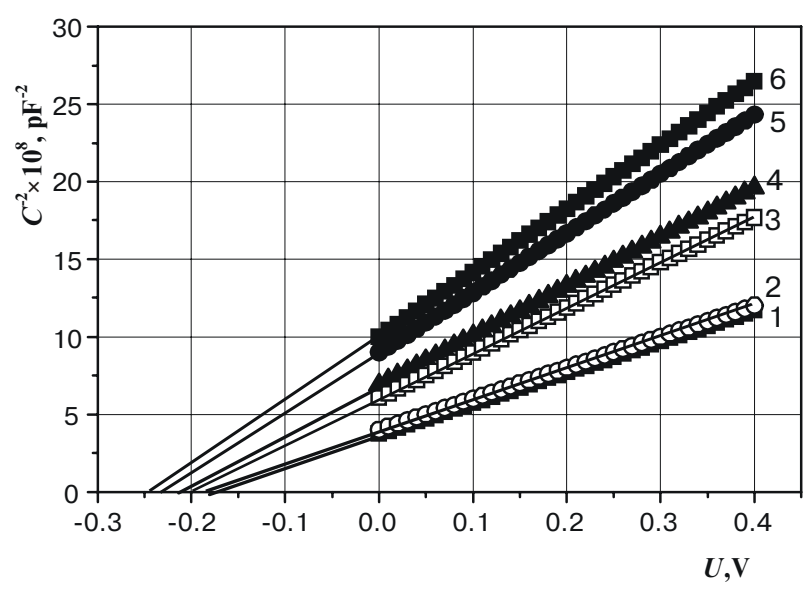

Fig. 2. Barrier capacitance $C^{-2}$ as a function of bias voltage $U$ in $\mathrm{Cu} / \mathrm{p}-\mathrm{PbTe}$ Ga contact measured at temperatures, K: 4.2 (1), 37 (2), 61 (3), 78 (4), 119 (5), 137 (6). The hole concentration is $8.3 \cdot 10^{16} \mathrm{~cm}^{-3}$ at $78 \mathrm{~K}$. Solid lines are calculated from equation (1) using the dielectric constant as fitting parameter. 


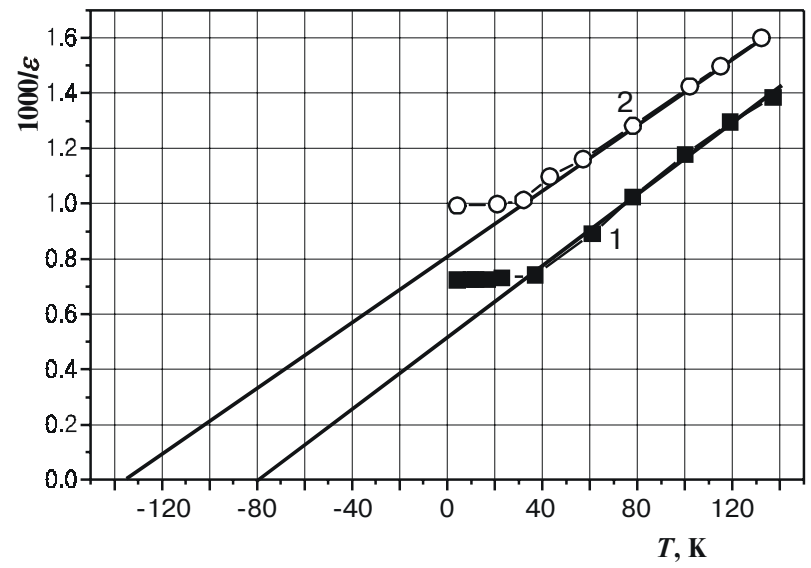

Fig.3. Reciprocal value of the static dielectric constant as a function of temperature in the samples of $\mathrm{Cu} / \mathrm{p}-\mathrm{PbTe}: \mathrm{Ga}$ contacts. At $78 \mathrm{~K}$ the hole concentration in the samples is $8.3 \cdot 10^{16} \mathrm{~cm}^{-3}(1)$ and $8.7 \cdot 10^{17} \mathrm{~cm}^{-3}(2)$.

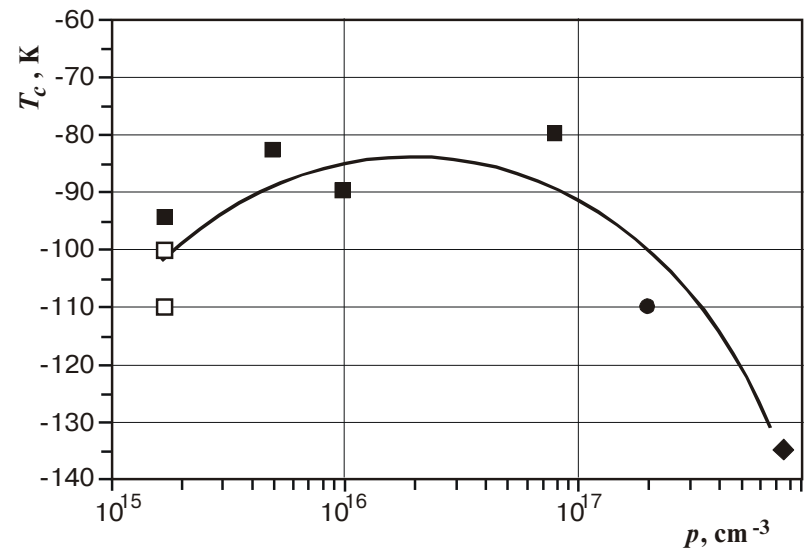

Fig. 4. The Curie temperature as a function of the hole concentration in the samples of the Schottky contacts.Open and close dots refer to the contacts $\mathrm{In} / p-\mathrm{PbTe}$ and $\mathrm{Cu} / p-\mathrm{PbTe}$, respectively.

obtained. The Curie temperature is nonmonotonous function of the carrier concentration. At $p \leq 10^{17} \mathrm{~cm}^{-3}$ the tendency to saturation or even small decreasing of the Curie temperature is observed. Other obvious feature is that the Curie temperature in compensated substrates has lower values in comparison with undoped $\mathrm{PbTe}$ when the concentration of free carriers is comparable (see, for instance, results of capacitance measurements in undoped $\mathrm{PbTe}$ [12]). The experimental value of $T_{c}$ in undoped $\mathrm{PbTe}$ with $p \cong 8 \times 10^{17} \mathrm{~cm}^{-3}$ is close to the values obtained previously $[2,3,5,12]$.

Different theoretical models have been proposed for explanation of paraelectric properties of IV-VI narrowgap semiconductors. Today, the model of interband electron-phonon coupling started by Konsin and Kristofell
$[10,15]$ and developed by other authors $[2,3,5,16,17]$ seems to be the most useful. In the framework of this model the dependence of the TO-phonon softening and Curie temperature on the concentration of free carriers in $\mathrm{Pb}_{1-\mathrm{x}} \mathrm{Sn}_{\mathrm{x}} \mathrm{Te}$ alloys has been theoretically analyzed in several studies $[2,3,5,16,17]$. The renormalized TOphonon frequency caused by interband electron-phonon coupling is given by [17]

$$
\omega_{T}^{* 2}=\omega_{T}^{2}-\frac{4 D^{2}}{M a^{2} W} \ln \frac{W}{2 E_{F}+E_{G}}
$$

where $W$ is the sum of the conduction and the valence bands width, $D$ is the optical deformation potential, $M$ is the reduced masses of ions in the unit cell, $a$ is the lattice constant and $E_{F}$ is the Fermi energy measured from the band edge, $\omega_{T}$. is the frequency of uncoupled TO-phonon.

The Curie temperature can be calculated from the equation [16]

$4 k T_{c}=\gamma-2 E_{F}-E_{G}$,

where

$\gamma=W \exp \left(-\frac{M a^{2} W \hbar^{2} \omega_{T}^{2}}{4 D^{2}}\right)$

Some remarks should be made concerning the calculation of the Curie temperature. There has been discussion of the values of the deformation potential obtained from different measurement techniques [5]. Its value known in literature is ranged from $0.5 \mathrm{eV}$ up to $20 \mathrm{eV}$. The Curie temperature of the order of $100 \mathrm{~K}$ may be obtained for the values of $W=2.5 \mathrm{eV}, D=1.43 \mathrm{eV}$ and $\hbar \omega_{T}=0.0039 \mathrm{eV}$ which were used in [16]. For comparison, in [17] TO-phonon frequency $\omega_{T}$ was calculated for the values $D=5 \mathrm{eV}$ and $W=10 \mathrm{eV}$. Despite large spread in the values of $D$ and $W$ the model correctly predicts behavior of the Curie temperature in undoped $\mathrm{PbSnTe}$ and $\mathrm{SnTe}$ compounds with degenerate gas of free carriers. As follows from the equations (2-4), occupancy of the conduction or valence bands by free carriers results in increase of the effective energy for interband electons transitions and decrease of the Curie temperature. Shown in Fig. 4 decreasing of $T_{c}$ at $p>10^{17} \mathrm{~cm}^{-3}$ is in accordance with theoretical prediction. However, its behavior at $p \leq 1 \times 10^{17} \mathrm{~cm}^{-3} \mathrm{can}$ not be explained in the framework of this model.

The influence of impurities on the Curie temperature is well known in wide-gap semiconductors [18]. In doped with foreign impurities crystals, the Curie temperature shifts towards the negative temperatures in comparison with the undoped (pure) ones. In accordance with the phenomenological theory of the structural phase transitions, this shift may be attributed both to free and localized carriers. The role of the localized carriers is especially important in high-resistance compensated semiconductors [18]. Moreover, the value of the shift linearly depends on the concentration of the localized carriers. 


\section{Tetyorkin, S. Movchan: Paraelectric properties of PbTe doped with Ga}

Experimental data shown in Fig. 4 may be explained in assumption that the doping with $\mathrm{Ga}$ is accompanied by introduction of deep states in the gap. To make predominant influence on the Curie temperature, their density should exceed much more the concentration of the holes in the valence band. The pronounced freeze-out of the holes and electrons on the states in the gap was observed in doped PbTe:Ga crystals [19].

An alternative approach is associated with deep defect centers in lead telluride which exhibit lattice relaxation due to Jahn-Teller effect. The Jahn-Teller center was proposed by Volkov and Pankratov for Te vacancy in $\mathrm{PbTe}$ [20]. Because of lattice symmetry around the JanhTeller center is changed when its charge state is altered, their contribution to the phase transition may be appreciable in highly doped crystals. An experimental evidence of the phase transition caused by impurity has been found in PbSnTe crystals highly doped with indium [21].

\section{Conclusions}

In summary, barrier capacitance has been investigated in the Schottky contacts prepared on compensated $p$ PbTe:Ga substrates. It is shown that compensation affects the dielectric constant of PbTe significantly. The increase of the compensation shifts the Curie temperature to lower negative values. Despite the fact that this experimental result is in accordance with phenomenological theory of the phase transition, exact mechanism of this shift in PbTe is not known clearly today. The influence of the impurity on the Curie temperature due to the Jahn-Teller effect may be also important.

\section{References}

1. R. Dornhaus, G. Nimitz and B. Schlicht, Narrow-Gap Semiconductors, Springer (1985).

2. H. Kawamura, Comments Solid State Phys.,9, 55 (1979).

3. H. Kawamura, S. Takano, K. Murase, S. Katayama, S. Nishi and S. Hotta, Prog. Theor. Phys, Suppl. 57, 156 (1975).

4. G. Bauer, in Lecture Notes in Phys., 77, 259 (1983).

5. W.J. Daughton and B. DeFacio, J. Phys. C., 11, 4307 (1978).

6. H. Heinrich, in Proc. III Summer Sch., Nimes, 407 (1979).

7. N.J.Parada, G.W.Pratt, Phys. Rev. Lett., 22, 180 (1969).

8. C.R.Hewes, M.S.Adler, and S.D.Senturia, Appl. Phys. Lett., 44, 1327 (1973).

9. A. Lopez-Otero, Thin. Solid Films, 49, 3 (1978).

10. N.N. Kristoffel and P.I. Konsin, Fiz. Tverd. Tela 13, 3513 (1971).

11. V.M. Lakeenkov, V.V. Tetyorkin, F.F. Sizov, S.V. Platsko and S.A. Belokon', Ukr. Fiz. Zhurnal, 18, 1608 (1984).

12. W. Jantsh and A. Lopez-Otero, in Proc. 13th Conf. Phys. Semicond, Rome, 487 (1976).

13. F.F.Sizov, V.V.Tetyorkin, Yu.G.Troyan and V.Yu.Chopick. Infrared Phys., 29, 271 (1989).

14. H. Rhoderick, Metal-Semiconductor Contacts, Clarendon, Oxford (1978).

15. N. Kristoffel, P. Konsin, Ferroelectrics, 6, 3 (1973).

16. V.L.Volkov, V.I.Litvinov, V.M.Baginskii and K.D.Tovstyuk. Fiz. Tverd. Tela 18, 3670 (1976).

17. H. Kawamura, S. Katayama, S. Takano and S. Hotta, Sol. State Commun., 14, 259 (1974).

18. V.M.Fridkin, Segnetoelektriki-poluprovodniki, «Nauka», Moscow (1976).

19. F.F. Sizov, Platsko S.V., Lakeenkov V.M., Fiz. Tehnika. Poluprovod., 19, 592 (1985), (in Rassian).

20. B.A. Volkov and O.A. Pankratov, Doklad. Akad. Nauk, 255, 93 (1980).

21. K.H. Herrmann and K.P Mollman, phys. stat. sol. (a), 80, K101 (1983). 